\title{
Solução nutritiva para produção de menta em hidroponia
}

\author{
Dalva Paulus ${ }^{1 ; 3}$; Sandro L. P. Medeiros²; Osmar S. Santos²; Eloi Paulus² \\ ${ }^{1}$ USP-ESALQ, Depto. Eng. Rural, 13418-900 Piracicaba-SP; ${ }^{2}$ UFSM, Depto. Fitotecnia, 97105-900 Santa Maria-RS; $97105-900$ Santa \\ Maria RS; ${ }^{3}$ End. para corresp.: Univ. Tecnol. Fed. do Paraná, C. Postal 157, 85660-000 Dois Vizinhos-PR; \\ dalvaufsmdeutch@yahoo.com.br
}

\section{RESUMO}

A menta (Mentha arvensis L.) é uma planta aromática e que produz um óleo essencial rico em mentol. Para o cultivo hidropônico de hortaliças folhosas e de frutos existe um grande número de soluções nutritivas recomendadas pela pesquisa; no entanto, existem poucas informações sobre soluções nutritivas para menta. O objetivo deste trabalho foi avaliar uma solução nutritiva para o cultivo de menta em hidroponia. Conduziram-se dois experimentos de outubro a dezembro de 2004 em Santa Maria, RS. Para o experimento a campo, as mudas foram obtidas por estacas e produzidas em substrato organomineral (plantmax ${ }^{\circledR}$ ), posteriormente transplantadas para canteiros de 5,00 m de comprimento e 1,00 de largura, no espaçamento 0,60 x 0,30 m. No sistema hidropônico "NFT", as mudas foram propagadas por estaquia em espuma fenólica, onde permaceram 18 dias no berçário, quando foram transferidas para as bancadas de produção final. O delineamento experimental foi blocos ao acaso em esquema fatorial 2x2 com os fatores: concentração da solução nutritiva e espaçamentos. Determinou-se a fitomassa seca, o teor de óleo essencial e o mentol. Os melhores resultados de teor de óleo essencial

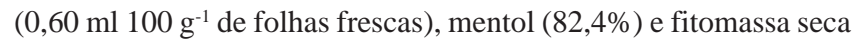
(45 g planta $^{-1}$ ) foram obtidos com 100\% da concentração da solução nutritiva no transplante e reposição de $50 \%$ quando a condutividade elétrica reduziu $50 \%$ do valor inicial e no espaçamento de $0,50 \mathrm{~m} \mathrm{x}$ $0,25 \mathrm{~m}$ nos canais de cultivo. Constatou-se que o teor e rendimento de óleo essencial e fitomassa seca no cultivo hidropônico com a solução nutritiva proposta foram superiores aos encontrados em cultivo a campo de $27 \mathrm{~g}$ planta-1 $^{-1} \mathrm{de}$ fitomassa seca, 0,53 $\mathrm{Ml} 100 \mathrm{~g}^{-1} \mathrm{de}$ folhas frescas de óleo essencial e 64,43\% de mentol. A solução nutritiva com concentração de $100 \%$ no transplante e reposição de $50 \%$ dos nutrientes quando a condutividade elétrica reduzir $50 \%$ do valor inicial e o espaçamento $0,50 \times 0,25 \mathrm{~m}$ pode ser recomendada para o cultivo hidropônico de Mentha arvensis.

Palavras-chave: Mentha arvensis L., nutrientes, cultivo hidropônico.

\begin{abstract}
Nutrient solution for mint production in hydroponic solution

The mint (Mentha arvensis L.) is an aromatic plant that produces essential oil with high menthol concentration. For the hydroponic cultivation of vegetables and fruits, a great number of recommended formulas exist; however, little information exists about hydroponic solutions for mint. The goal of this work was to evaluate a nutrient solution for the mint cultivation in hydroponic system. Two experiments were carried out from October to December, 2004. For the field experiment, transplants were obtained from cuttings and produced in organic mineral substrates (plantmax ${ }^{\circledR}$ ). Later they were transplanted in seed beds of $5.00 \mathrm{~m}$ length and $1.00 \mathrm{~m}$ width, spacings of $0.60 \times 0.30 \mathrm{~m}$. For the hydroponic system NFT, seedlings were obtained from cuttings and planted in phenolic foam where they stayed during 18 days. After that, they were transplanted to a production canal. The experimental design was a $2 \times 2$ factorial (nutrient solution concentrations $\mathrm{x}$ spacings). The hydroponic solution with $100 \%$ concentration in transplant and 50\% replacement when the electric condutivity decreased $50 \%$ of the initial value and $0.5 \times 0.25 \mathrm{~m}$ spacings resulted in the highest concentration of essential oil (0.60 mL $100 \mathrm{~g}^{-1}$ fresh leaves), menthol (82,4\%) and total dry matter (45 g plant $\left.^{-1}\right)$. In hydroponic system the yield of dry matter and content of essential oil was higher than under soil conditions 27 g planta $^{-1}$ of dry matter, concentration oil $0.53 \mathrm{~mL} 100 \mathrm{~g}^{-1}$ and $64,43 \%$ of menthol. The hydroponic solution with $100 \%$ concentration in transplant and 50\% replacement when the electric condutivity decreased $50 \%$ of the initial value and $0.5 \times 0.25 \mathrm{~m}$ spacing can be recommended for the cultivation of mint in hydroponic solution.
\end{abstract}

Keywords: Mentha arvensis, nutrients, hydroponic cultivation.

\section{(Recebido para publicação em 28 de março de 2007; aceito em 21 de fevereiro de 2008)}

\begin{abstract}
A menta ou hortelã japonesa (Mentha arvensis L.) pertence à família Lamiaceae, sendo que no Brasil a principal cultivar plantada é IAC 701 (Maia, 1998). É uma planta aromática, com folhas e inflorescências ricas em óleo essencial, sendo que o mentol é o seu principal e mais abundante componente. $\mathrm{O}$ óleo essencial da menta é empregado na indústria alimentícia, farmacêutica e de higiene. Na medicina popular a menta é utilizada no tratamento de distúrbios digestivos e de verminoses. Essas características lhe conferem grande importância econômica (Martins et al., 1994).
\end{abstract}

A menta é considerada planta exigente em nutrição (Maia, 1998), preferindo solos férteis, bem drenados e ricos em matéria orgânica (Correa Jr. et al., 1991).

O Brasil foi o principal produtor mundial de óleo de menta até o final da década de 70, quando ocorreu uma redução drástica da sua produção a campo, devido ao monocultivo, o que desencadeou em problemas de fertilidade, como baixo teor de matéria orgânica, deficiência nutricional de macro e micronutrientes (Maia, 1994).
A recuperação da posição brasileira no mercado de óleo essencial depende da retomada da produção de menta. Nesse sentido, a hidroponia tem se tornado uma alternativa bastante interessante em relação ao cultivo tradicional feito no solo. Podem-se destacar algumas vantagens da hidroponia: alta produção/área, maior controle no fornecimento de nutrientes e obtenção de produtos de alta qualidade (Castellane \& Araújo, 1995). Além disso, sua utilização em ambiente protegido permite cultivos durante o ano todo. Plantas aromáticas produzidas em sistema 
hidropônico apresentam elevada produtividade e três a seis vezes mais óleo essencial por planta do que em sistemas convencionais de cultivo (Mairapetyan, 1999).

Um dos aspectos mais importantes no cultivo de plantas em hidroponia é a solução nutritiva. Esta deve ser formulada de acordo com o requerimento da espécie que se deseja produzir, ou seja, conter proporções adequadas de todos os nutrientes essenciais ao desenvolvimento das plantas. O manejo de forma incorreta provoca diminuição na produtividade e na qualidade visual, que compromete a comercialização do produto e pode levar à perda completa da produção (Schmidt, 1999). O método que prevê a renovação completa da solução sem que haja a reposição dos nutrientes e renovação completa da solução, apresenta inconvenientes como o desperdício de água e sais e o efeito poluente ao meio ambiente (Londero, 2000). De acordo com o autor, o método de reposição de nutrientes com base na redução de $50 \%$ da condutividade elétrica inicial apresentou melhor desempenho em cultivo de alface com solução nutritiva Castellane \& Araújo (1995).

As concentrações de nutrientes para elaboração de soluções nutritivas para o cultivo de menta, de acordo com Barry (1996), seguem as seguintes faixas (mg $\left.\mathrm{L}^{-1}\right)$ : N (70-250), P (15-80), K (150-400), Ca (70-200), Mg (15-80), S (20-200), Fe (0,8-6), Mn (0,5-2), B (0,1-0,6), Cu $(0,05-0,3)$, Zn $(0,1-0,5)$ e Mo $(0,05-$ $0,15)$.

Na definição de uma solução nutritiva busca-se uma formulação de concentração de nutrientes que atenda às exigências nutricionais da cultura e resulte em elevada produção de fitomassa e óleo essencial com qualidade (Maia, 1998). De acordo com o mesmo autor, concentrações de nitrogênio superiores a $420 \mathrm{mg} \mathrm{L}^{-1}$ reduziram a produção e qualidade do óleo. Por outro lado, a concentração de fósforo utilizada como referência no preparo de soluções nutritivas para esta cultura deve ser em torno de $31 \mathrm{mg} \mathrm{L}^{-1}$, pois concentrações maiores não melhoram a produtividade da planta nem a qualidade do óleo. Com relação ao cálcio, este elemento facilita a absorção de outros nutrientes, além de aumentar significativamente o teor de óleo nas folhas (Maia, 1998). Quanto ao magnésio, o autor recomenda concentração de $48 \mathrm{mg} \mathrm{L}^{-1}$ deste elemento pois, concentrações maiores reduzem o teor de mentol prejudicando a qualidade do óleo essencial devido a reduzir o teor de mentol. Já para o enxofre a concentração recomendada pelo autor é de 64 mg L ${ }^{-1}$ do nutriente.

Diversas formulações têm sido sugeridas para o cultivo hidropônico, as quais apresentam diferentes concentrações de nutrientes, $\mathrm{pH}$ e condutividade elétrica, de acordo com a espécie a ser cultivada. O cultivo em condutividades elétricas inferiores às recomendadas pode resultar em deficiências múltiplas e retardo no crescimento das plantas (Martinez \& Silva Filho, 1997).

Há grande número de fórmulas recomendadas para o cultivo de hortaliças. No entanto, existem poucas informações com relação ao comportamento da menta nessas soluções. Maia (1998), em trabalhos com solução nutritiva proposta por Sarruge (1975), relata que a concentração dos nutrientes utilizada não se mostrou eficiente na produção de biomassa e óleo essencial. Segundo o autor, pesquisas devem ser desenvolvidas para se definir as concentrações de nutrientes adequadas na solução nutritiva para o cultivo de menta em hidroponia. O objetivo deste trabalho foi propor e avaliar uma solução nutritiva para o cultivo de menta em hidroponia.

\section{MATERIAL E MÉTODOS}

Foram conduzidos dois experimentos de outubro a dezembro de 2004. Um dos experimentos foi conduzido em hidroponia, em estufa plástica de 250 $\mathrm{m}^{2}$ coberta com cloreto de polivinil (PVC) com 200 ì de espessura. O outro experimento foi conduzido a campo ( $29^{\circ} 42^{\prime} \mathrm{S}$ de latitude, $53^{\circ} 43^{\prime} \mathrm{W}$ de longitude e $95 \mathrm{~m}$ de altitude). O solo da área experimental é classificado como Alissolo Hipocrômico Argilúvio típico (Embrapa, 1999).

O delineamento experimental foi de blocos ao acaso com oito repetições em esquema fatorial $2 \mathrm{x} 2$, com os fatores: concentração da solução nutritiva: A)
100\% da concentração da solução no transplante, com reposição de $50 \%$ dos nutrientes quando a condutividade elétrica reduziu a $50 \%$ do valor inicial (SC) B) $25 \%$ da concentração da solução no transplante, com reposição de $25 \%$ da solução a cada 15 dias (SCP) e espaçamentos: A) $0,50 \times 0,25 \mathrm{~m}$, correspondendo a 8 plantas $\mathrm{m}^{-2}$ (D8); B) 0,25 x 0,25m, correspondendo a 16 plantas $\mathrm{m}^{-2}$ (D16).

Coletaram-se amostras de solo que foram analisadas em laboratório da UFSM, obtendo-se: $\mathrm{pH}$ em água (1:1)= 5,5; P, K, Cu e Zn (mg..$\left.^{-1}\right)=48,5 ; 200$; 2,2 e 3,5; matéria orgânica $=2,1 \%$; saturação de bases=60\%; $\mathrm{Al}, \mathrm{Ca}, \mathrm{Mg}$ e CTC efetiva $\left(\mathrm{cmol}_{c} \mathrm{l}^{-1}\right)=0,0 ; 3,5 ; 1,4 \mathrm{e}$ 5,4 . Não foi necessário efetuar adubação de base, porque os resultados de análise de solo atendiam aos padrões da Comissão de Fertilidade do Solo (1994).

As mudas de menta japonesa (Mentha arvensis) cultivar IAC 701, para plantio em campo, foram obtidas de estacas de aproximadamente $7 \mathrm{~cm}$ de comprimento e com quatro folhas, colocadas para enraizar em caixas de madeira medindo $0,35 \mathrm{~m}$ de largura $\mathrm{x}$ 0,75 $\mathrm{m}$ de comprimento, contendo substrato organo-mineral (Plantmax ${ }^{\circledR}$ ). Estas permaneceram neste local até o momento que apresentaram 7 a 8 folhas completamente desenvolvidas, aos 20 dias, quando foram transplantadas para canteiros de $5 \mathrm{~m}$ de comprimento e $1 \mathrm{~m}$ de largura, no espaçamento de 0,6 x 0,3 m correspondendo à densidade de 5 plantas $/ \mathrm{m}^{2}$, recomendado por Maluf et al. (1999). Foram realizadas irrigações com mangueira, diariamente nos períodos mais secos. Realizou-se uma adubação em cobertura 20 dias após o transplante utilizando-se $30 \mathrm{~kg} \mathrm{ha}^{-1}$ de $\mathrm{N}$ e $25 \mathrm{~kg} \mathrm{ha}^{-1}$ de $\mathrm{K}_{2} \mathrm{O}$.

As mudas de menta japonesa, cultivar IAC 701, que seriam transplantadas no experimento em hidroponia, foram obtidas de matrizes produzidas em sistema hidropônico na UFSM e propagadas por estaquia. Utilizou-se estacas de aproximadamente $3,5 \mathrm{~cm}$ de comprimento e com quatro folhas, colocadas para enraizar em substrato espuma fenólica, sendo conduzidas em berçário constituído de tubos de polipropileno, com $4 \mathrm{~m}$ de comprimento e $3 \mathrm{~cm}$ de lar- 
gura, colocados sobre cavaletes com desnível de 1\% para o escoamento da solução nutritiva. As mudas permaneceram no berçário até o momento que apresentaram sete a oito folhas completamente desenvolvidas, aos 18 dias, quando foram transplantadas para a bancada de produção final.

A bancada de produção final foi constituída de seis canais de cultivo de polipropileno com $6 \mathrm{~m}$ de comprimento e $10 \mathrm{~cm}$ de largura. Os canais de cultivo foram sustentados por cavaletes de madeira de 0,80 m de altura, com declividade de $2 \%$. Utilizou-se dois espaçamentos (entre canais e entre plantas): $0,50 \times 0,25 \mathrm{~m}$ e $0,25 \times 0,25 \mathrm{~m}$, correspondendo à densidade de oito e 16 plantas $\mathrm{m}^{-2}$, respectivamente. A solução nutritiva foi calculada a partir dos dados de produção de fitomassa seca e da quantidade de nutrientes extraídos pela planta, obtidos por Maia (1994). Para o preparo da solução nutritiva utilizou-se os nutrientes (mg $\left.\mathrm{L}^{-1}\right): \mathrm{K}^{+}=$ 299,52; $\mathrm{Ca}^{++}=79,60 ; \mathrm{Mg}^{++}=34,80 ; \mathrm{N}-$ $\mathrm{NO}_{3}^{-}=211,40 ; \mathrm{P}_{-} \mathrm{H}_{2} \mathrm{PO}_{4}^{-}=27,90 ;{\mathrm{S}-\mathrm{SO}_{4}^{-}}_{4}^{-}$ 11,$84 ; \mathrm{B}=2,844 ; \mathrm{Cu}=0,075 ; \mathrm{Mn}=$ 6,$084 ; \mathrm{Mo}=0,0883 ; \mathrm{Zn}=0,431 ; \mathrm{FeSO}_{4}^{-}=$ 6,$08 ; \mathrm{Na}_{2} \mathrm{EDTA}=14,890$. Para produção de mudas, esta foi diluída a 50\% da concentração original, conforme Furlani (1998). As fontes de nutrientes empregadas e as quantidades (g $1000 \mathrm{~L}^{-1}$ ) da solução nutritiva, respectivamente, foram: $\mathrm{Ca}\left(\mathrm{NO}_{3}\right)_{2} \cdot 4 \mathrm{H}_{2} \mathrm{O}=398,00 ; \mathrm{KNO}_{3}=$ 820,36; $\mathrm{MgNO}_{3}=274,32 ; \mathrm{NH}_{4} \mathrm{H}_{2} \mathrm{PO}_{4}=$ 145,24; $\mathrm{MgSO}_{4} .7 \mathrm{H}_{2} \mathrm{O}=91,08 ; \mathrm{H}_{3} \mathrm{BO}_{3}=$ 2,844; $\mathrm{CuSO}_{4} .5 \mathrm{H}_{2} \mathrm{O}=0,075$; $\mathrm{MnSO} 4 . \mathrm{H} 2 \mathrm{O}=6,084 ; \mathrm{ZnSO}_{4} \cdot 7 \mathrm{H}_{2} \mathrm{O}=$ 0,431; $\left(\mathrm{NH}_{4}\right)_{6} \mathrm{Mo}_{7} \mathrm{O}_{24} \cdot 4 \mathrm{H}_{2} \mathrm{O}=0,0883$; $\mathrm{Fe}-\mathrm{EDTA}=500 \mathrm{~mL}$.

Em função da curva de crescimento da cultura de Maia (1994) testou-se na cultura da menta japonesa um tratamento com concentração parcelada de solução nutritiva, o qual foi de $25 \%$ da concentração original no transplante, com reposição fixa de $25 \%$ da solução a cada 15 dias, repondo-se a solução aos 15 e 30 dias após transplante. O outro tratamento consistiu de $100 \%$ da concentração da solução no transplante, com reposição de 50\% dos nutrientes quando a condutividade elétrica reduziu a 50\% do valor inicial,o que ocorreu aos 18, 22 e 28 dias no ciclo.
Os micronutrientes da fórmula foram fornecidos na forma de solução concentrada, obtida através da dissolução separada dos sais em 100mL de água. Após a diluição dos sais, completou-se o volume para um litro. Como fonte de ferro utilizou-se Fe-EDTA, obtido através da dissolução de 6,08 g de sulfato de ferro que foram diluídas em $500 \mathrm{~mL}$ de água e 14,89 g de $\mathrm{Na}_{2}$-EDTA em 400 $\mathrm{mL}$ de água quente $\left(80^{\circ} \mathrm{C}\right)$. Após o resfriamento do $\mathrm{Na}_{2}$-EDTA, misturouse as duas soluções, completando o volume para 1 litro e borbulhando ar por 12 horas, no escuro, conforme recomendação de Martinez \& Silva Filho (1997).

A condutividade elétrica inicial das soluções 25\% e 100\% foram de 0,56 dS/ m e $1,99 \mathrm{dS} / \mathrm{m}$. O controle da circulação da solução nutritiva foi realizado com o auxilio de um temporizador programado para acionar a moto-bomba durante $15 \mathrm{~min}$, com intervalos de 15 min, no período diurno (7 h-20 h), e 15 min a cada intervalo de 2 h no período noturno (20 h-7 h).

$\mathrm{O}$ pH das soluções nutritivas foi corrigido a cada dois dias no período da

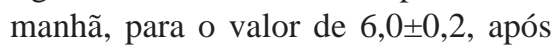
completar o volume dos reservatórios com água. Utilizou-se hidróxido de sódio $(\mathrm{NaOH}$ 0,3 N) ou ácido sulfúrico $\left(\mathrm{H}_{2} \mathrm{SO}_{4} 10 \%\right)$ para elevar ou diminuir o pH. A leitura da condutividade elétrica da solução nutritiva manteve a mesma frequência das leituras de $\mathrm{pH}$.

Realizaram-se coletas de plantas a cada sete dias para determinação do acúmulo de massa seca. A colheita do experimento em hidroponia e a campo foi realizada 35 e 67 dias, respectivamente, após o transplante quando 70\% dos botões florais estavam na eminência de abrirem, conforme recomendado por Singh \& Singh (1989). As plantas coletadas foram separadas em raíz, hastes e folhas, determinando-se a fitomassa fresca aérea das hastes e folhas. Posteriormente, o material coletado foi colocado em estufa de secagem com ventilação forçada de ar a $65^{\circ} \mathrm{C}$, até massa constante, para determinação da massa seca e análise de tecidos.

Para extração do óleo essencial do material a campo e em hidroponia foi utilizado o método de hidrodestilação, em aparelho do tipo Clevenger. Amos- tras de $100 \mathrm{~g}$ de fitomassa verde de folhas foram colocadas em balões com capacidade de dois litros, com período de destilação de 1 h e 20 min, conforme metodologia descrita por Alencar et al. (1984).

Os cromatogramas foram obtidos em cromatógrafo a gás Variam 3800 equipado com detector de ionização de chama (DIC), injetor "Split-Splitless”, operando com as temperaturas do injetor e do detector a $220^{\circ} \mathrm{C}$ e $280^{\circ} \mathrm{C}$ respectivamente. $\mathrm{O}$ gás de arraste utilizado foi $\mathrm{H}_{2}$ a $50 \mathrm{kpa}$ de pressão (1 mL/min). Foram empregadas colunas de capilares de sílica fundida (CCSF) com $25 \mathrm{~m}$ de comprimento e 32 mm de diâmetro, com fases estacionárias SE-54 (Polissiloxano) e programa de injeção de amostra com fluxo de 1 ìL, temperatura de $50-250^{\circ} \mathrm{C}$ e rampa de aquecimento igual a $4^{\circ} \mathrm{C} / \mathrm{min}$.

A identificação dos constituintes foi determinada através de comparação dos espectros de massa dos picos cromatográficos com os espectros CG/ MS (Cromatografia gasosa/espectros de massa) e com os padrões de igual índice de retenção encontrados por Adams (1995).

Os dados obtidos foram submetidos à análise de variância e foram ajustadas equações de regressão para as características de crescimento das plantas.

\section{RESULTADOS E DISCUSSÃO}

Verificou-se que as concentrações de nitrogênio, fósforo e magnésio, no preparo da solução nutritiva proposta, foram similares às propostas por Maia (1998). Para o potássio, cálcio e enxofre, as concentrações foram inferiores às concentrações citadas pelo autor. Desta forma, devido à menor quantidade de nutrientes utilizada, obteve-se economia desse insumo.

Comparando a quantidade de macronutrientes da solução nutritiva proposta com a solução nutritiva de Hoagland e Arnon (1950), as quantidades de $\mathrm{N}$ e $\mathrm{K}$ foram 1,4 $\mathrm{mg} \mathrm{L}^{-1}$ e 65,52 $\mathrm{mg} \mathrm{L}^{-1}$ superior, já as quantidades dos demais macronutrientes foram inferiores. Com relação aos micronutrientes, as quantidades foram similares. A quantidade de macro e micronutrientes da 
Tabela 1. Teor médio de mentol e mentona (\%) do óleo essencial das folhas de Mentha arvensis L., cultivar IAC 701, cultivada em solução nutritiva nas concentrações: 100\% (SC); 25\% (SCP) e dois espaçamentos: 0,50 x 0,25 m (D8); 0,25 x 0,25 m (D16). (Medium content of menthol and mentona (\%) on leaves of Mentha arvensis L., cultivar IAC 701, cultivated in hidroponic solution in the concentrations $100 \%$ (SC) and $25 \%$ (SCP), and two spacings: 0,50 x 0,25 m (D8); 0,25 x 0,25 m (D16)). Santa Maria, UFSM, 2004.

\begin{tabular}{lcccc}
\hline \multirow{2}{*}{ Substâncias } & \multicolumn{4}{c}{ Tratamentos } \\
\cline { 2 - 5 } & SC & SCP & Espaçamentos & C.V.\% \\
\hline \multirow{2}{*}{ Mentol } & $82,4 a^{*}$ A & 67,05 a B & D8 & \multirow{2}{*}{10,02} \\
& 71,5 b A & 64,20 a B & D16 & \multirow{2}{*}{ Mentona } \\
\hline \multirow{2}{*}{ 8,8 a B } & 19,70 a A & D8 & 19,98 \\
\hline
\end{tabular}

*Médias seguidas das mesmas letras, minúsculas nas colunas e maiúsculas nas linhas, em cada variável, não diferem pelo teste de F (pd”0,05). (Means followed by the same small letters in the column and capital in the line did not differ through the F test (pd”0,05).

solução nutritiva proposta foi elaborada para atender as exigências nutricionais da espécie e resultar em maior produção e qualidade de óleo essencial.

No presente trabalho não foram observados sintomas de deficiência nutricional com as concentrações de nutrientes utilizadas na solução nutritiva durante o cultivo, sendo que, a análise de tecidos revelou que o potássio foi o nutriente encontrado em maior quantidade na planta (Tabela 2). As concentrações de nutrientes utilizadas no preparo da solução nutritiva estão de acordo com o intervalo de concentração de nutrientes recomendados por Maia (1998) e Barry (1996).
O rendimento de massa seca das folhas apresentou resposta quadrática em relação às soluções SC e SCP e espaçamento D8 (Figura 1). No espaçamento D16, as equações que melhor se ajustaram foram linear para SC e quadrática para SCP. A produção total das plantas ocorreu aos 35 DAT, que correspondeu com o auge da floração, período recomendado para colheita e extração do óleo. Neste estudo, a produção de fitomassa apresentou comportamento diferenciado em relação às concentrações da solução nutritiva e espaçamento. Sendo superior no tratamento SC-D8 (22,09 g planta $\left.^{-1}\right)$, em relação a SCP (7,86 g planta- $\left.{ }^{-1}\right)$. O mesmo se verificou no espaçamento D16, onde se obteve rendimento de 12,73 g planta ${ }^{-1}$ para SC e 7,06 g planta ${ }^{-1}$ para SCP.

Com relação à massa seca das hastes e total, a resposta das soluções nos diferentes espaçamentos foi quadrática, obtendo-se produção total superior de 45 g planta $^{-1}$ na SC e D8 (Figura 1).

Nas condições experimentais do estudo, os melhores resultados de produção das plantas foi observado utilizando-se o manejo com $100 \%$ da concentração da solução nutritiva no transplante e, com reposição de $50 \%$ dos nutrientes quando a condutividade elétrica reduziu a $50 \%$ do valor inicial (SC). O menor rendimento foi encontrado no tratamento com $25 \%$ da concentração da solução nutritiva no transplante, com reposição fixa de $25 \%$ da solução a cada 15 dias (SCP), pois esta foi menos concentrada em termos de nutrientes para atender às exigências nutricionais da cultura, verificando-se inclusive pelo baixo acúmulo de nutrientes na planta (Tabela 2). Tal fato acentuou-se com o adensamento de plantas no espaçamento $0,25 \times 0,25 \mathrm{~m}$, onde $\mathrm{o}$ adensamento resultou em maior competição entre plantas por água e nutrientes resultando em rendimento inferior ao espaçamento $0,50 \times 0,25 \mathrm{~m}$.

No cultivo a campo, com densidade de cinco plantas $\mathrm{m}^{-2} \mathrm{o}$ rendimento total de massa seca foi de $27 \mathrm{~g} \mathrm{planta}^{-1}$ num ciclo produtivo de 67 dias.

Tabela 2. Acúmulo de nutrientes nas raízes, hastes, folhas e planta inteira de menta (Mentha arvensis L.) cultivar IAC 701 cultivada em solução nutritiva nas concentrações de $100 \%$ (SC) e $25 \%$ (SCP) no espaçamento 0,50 x 0,25 m (D8). (Accumulation of nutrients in the roots, stems, leaves and entire plants of mint (Mentha arvensis L.), cultivar IAC 701, cultivated in hydroponic solution in the concentrations 100\% (SC) and 25\% (SCP) and spacing: 0,50 x 0,25 m (D8)). Santa Maria, UFSM, 2004.

\begin{tabular}{|c|c|c|c|c|c|c|c|c|}
\hline \multirow{2}{*}{ Nutr. } & \multicolumn{2}{|c|}{ Raizes } & \multicolumn{2}{|c|}{ Hastes } & \multicolumn{2}{|c|}{ Folhas } & \multicolumn{2}{|c|}{ Total } \\
\hline & SC & SCP & SC & SCP & SC & SCP & SC & SCP \\
\hline $\mathrm{N}^{* *}$ & $87,7 a^{*}$ & $33,1 \mathrm{~b}$ & 303,6 a & $88,2 \mathrm{~b}$ & 493,6 a & $191,9 \mathrm{~b}$ & $885,0 \mathrm{a}$ & $313,2 b$ \\
\hline$P$ & $21,0 \mathrm{a}$ & $6,5 \mathrm{~b}$ & $42,2 \mathrm{a}$ & $15,8 \mathrm{~b}$ & 63,6 a & $23,4 \mathrm{~b}$ & $126,9 \mathrm{a}$ & $45,6 \mathrm{~b}$ \\
\hline K & $151,4 \mathrm{a}$ & $46,6 \mathrm{~b}$ & $499,0 \mathrm{a}$ & 206,8 b & $502,2 \mathrm{a}$ & $193,4 \mathrm{~b}$ & 1152,6 a & $446,9 \mathrm{~b}$ \\
\hline $\mathrm{Ca}$ & $39,5 \mathrm{a}$ & $12,6 b$ & $87,1 \mathrm{a}$ & $45,2 \mathrm{~b}$ & $172 a$ & $81,9 \mathrm{~b}$ & 298,6 a & $139,6 \mathrm{~b}$ \\
\hline $\mathrm{Mg}$ & $22,6 \mathrm{a}$ & $10,6 \mathrm{~b}$ & 34,3 a & $15,8 \mathrm{~b}$ & $79,1 \mathrm{a}$ & $28,9 \mathrm{~b}$ & $136,0 \mathrm{a}$ & $55,2 \mathrm{~b}$ \\
\hline$S$ & $41,1 \mathrm{a}$ & $10,3 \mathrm{~b}$ & $29,0 \mathrm{a}$ & $13,7 \mathrm{~b}$ & 99,76 & 31,98 & 169,86 & 55,88 \\
\hline $\mathrm{Fe}^{* * *}$ & 13343,2 a & $3955,5 b$ & $1584 \mathrm{a}$ & $892,5 \mathrm{~b}$ & 3096 a & $1131 b$ & $18023,2 \mathrm{a}$ & $5979 b$ \\
\hline $\mathrm{Mn}$ & $8211,2 \mathrm{a}$ & $923 b$ & $1188 a$ & $861 \mathrm{~b}$ & $2408 \mathrm{a}$ & $1170 \mathrm{~b}$ & $11807,2 \mathrm{a}$ & $2954 b$ \\
\hline $\mathrm{Cu}$ & $118 a$ & 87,9 b & 184,8 a & $105 b$ & 189,2 a & 109,2 b & $492 \mathrm{a}$ & $302,1 \mathrm{~b}$ \\
\hline $\mathrm{Zn}$ & 359,2 a & $410,2 \mathrm{~b}$ & $211,2 \mathrm{a}$ & $147 \mathrm{~b}$ & 412,8 a & $280,8 \mathrm{~b}$ & 983 a & $838 b$ \\
\hline B & $225,8 \mathrm{a}$ & $111,6 \mathrm{~b}$ & $565 a$ & $210 b$ & 596,8 a & 249,6 b & 1387,6 a & 571,2 b \\
\hline
\end{tabular}

*Médias seguidas das mesmas letras nas linhas, em cada variável, não diferem pelo teste de F (pd”0,05). (Means followed by the same letters in the line did not differ through the F test (pd”0,05); **Macronutrientes expressos em mg por planta (Macronutrients expressed in $\mathrm{mg}$ in each plant); ${ }^{* * *}$ Micronutrientes expressos em $\mu \mathrm{g}$ por planta (Micronutrients expressed in $\mu \mathrm{g}$ in each plant). 


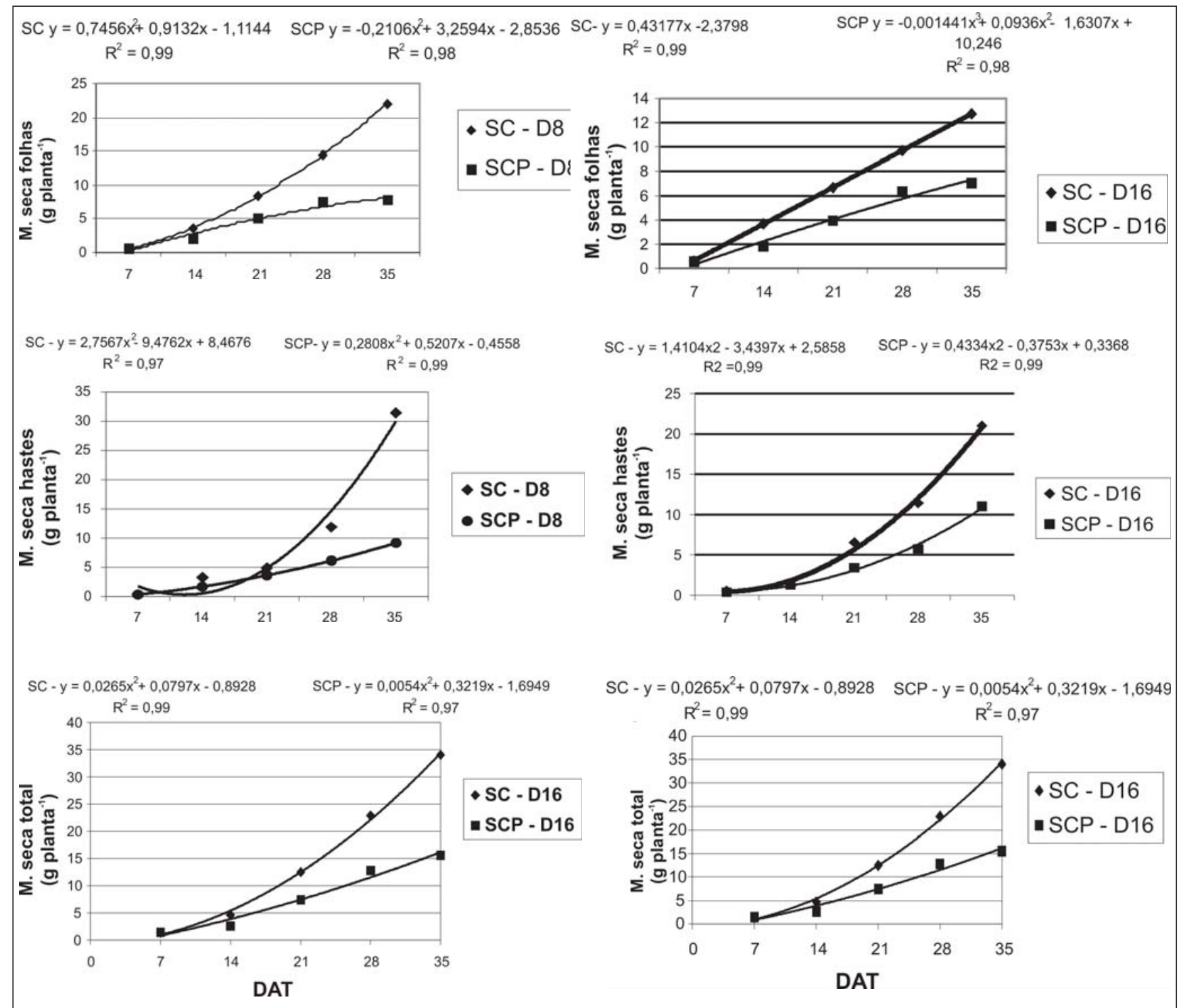

Figura 1. Produção de massa seca de folhas, hastes e total (g planta-1) de Mentha arvensis L., cultivar IAC 701, cultivada em solução nutritiva nas concentrações de 100\% (SC) e 25\% (SCP) em dois espaçamentos: 0,50 x 0,25 m (D8) e 0,25 x 0,25 m (D16). (yield of dry matter of leaves, stem and entire plants of mint (Mentha arvensis L.), cultivar IAC 701, cultivated in hydroponic solution in the concentrations 100\% (SC) and 25\% (SCP), and two spacings: 0,50 x 0,25 m (D8); 0,25 x 0,25 m (D16). Santa Maria, UFSM, 2004.

Com relação ao teor de óleo essencial, verificou-se que houve diferença significativa entre os tratamentos utilizados. Os melhores resultados para o teor de óleo essencial de folhas $(0,60 \mathrm{~mL}$ $100 \mathrm{~g} \mathrm{~g}^{-1}$ de folhas) foi observado quando as plantas foram cultivadas na solução nutritiva $100 \%$ da concentração da solução no transplante, com reposição de $50 \%$ dos nutrientes quando a condutividade elétrica reduziu a 50\% do valor inicial (SC) e espaçamento $0,50 \mathrm{x}$ $0,25 \mathrm{~m}$ (D8). O menor teor de óleo essencial nas folhas $\left(0,42 \mathrm{~mL} 100 \mathrm{~g}^{-1} \mathrm{de}\right.$ folhas) foi encontrado no tratamento com 25\% da concentração da solução nutritiva no transplante, com reposição de $25 \%$ da solução a cada 15 dias (SCP) e espaçamento 0,25 x 0,25 m (D16). Este tratamento, que apresentou menor rendimento de massa seca, também resultou em teor inferior de óleo. Assim, o maior rendimento de óleo essencial foi verificado no tratamento que apresentou maior produção de massa seca e ao mesmo tempo a maior concentração de óleo na massa seca. Um teor elevado de óleo por si só pode não ter interesse agrícola, pois plantas que produzam muito óleo, porém com baixa produção de folhas, resultarão em baixo rendimento de óleo por área.
O teor de óleo foi diferenciado nos sistemas de cultivo. No cultivo a campo obteve-se teor de óleo essencial de $0,53 \mathrm{~mL} 100 \mathrm{~g}^{-1}$ de folha fresca. Verificou-se que, no cultivo em hidroponia, o maior teor de óleo essencial foi de 0,60 $\mathrm{mL} 100 \mathrm{~g}^{-1}$ de folha fresca, obtido no tratamento SC-D8. Este valor foi de 0,12 $\mathrm{mL}$ superior em relação ao valor obtido a campo. Os resultados encontrados estão de acordo com os de Ocampos et al. (2002) em trabalhos com diversas formas de adubação (orgânica, mineral, orgânica/mineral e hidropônica) para hortelã rasteira (Mentha x villosa) obteve maior rendimento de massa fresca 
$\left(63,61 \mathrm{~g} \mathrm{planta}^{-1}\right)$ e de óleo essencial $\left(0,88\right.$ g planta $\left.^{-1}\right)$ no sistema de cultivo hidropônico.

Para o teor de mentol e mentona, observou-se concentrações diferenciadas em relação à solução nutritiva e espaçamento (Tabela 1). As plantas cultivadas na solução nutritiva com 100\% da concentração no transplante, com reposição de $50 \%$ dos nutrientes quando a condutividade elétrica reduziu a $50 \%$ do valor inicial (SC) e espaçamento 0,50 x 0,25 m (D8) apresentaram o maior teor de mentol nas folhas. Nas condições em que o experimento foi realizado, isso provavelmente se deve ao efeito da nutrição mineral sobre a composição de mentol no óleo essencial. Conforme Maia (1998), as condições de nutrição mineral alteram as proporções de mentol e mentona.

No experimento a campo obteve-se teor médio de mentol de 64,45\% e $17,67 \%$ de mentona. A percentagem de mentol encontrada nas folhas em campo foi inferior aos resultados obtidos nas plantas cultivadas em hidroponia com a solução nutritiva $100 \%$ da concentração no transplante, com reposição de 50\% dos nutrientes quando a condutividade elétrica reduziu a 50\% do valor inicial (SC) e espaçamento 0,50 x 0,25 m (D8). A hidroponia proporcionou melhores condições para o desempenho das plantas quanto ao rendimento de mentol quando comparadas em relação ao cultivo em solo. Resultados semelhantes foram obtidos por Maia (1998) em trabalhos de menta japonesa (Mentha arvensis) em vasos com solução nutritva proposta por Sarruge (1975) onde obteve o teor médio de mentol 82,36\% e de mentona 5,84\%.

Os teores de mentol encontrados no presente trabalho, tanto em hidroponia como em campo atendem as exigências da Farmacopéia Brasileira II, USNF 18, a qual preconiza um teor de mentol entre 30 e 55\% de mentol (Simões et al., 2003).

Observa-se na Tabela 2 que o acúmulo de nutrientes nas raízes, hastes, folhas e planta inteira de menta produzida na solução SC foi maior em relação ao tratamento com solução SCP. Verificou-se que houve maior concentração de nutrientes nas folhas, em seguida nas hastes e por último nas raízes.
O potássio, nitrogênio e cálcio foram os macronutrientes extraídos em maior quantidade tanto nas folhas como nas hastes e raízes. Os micronutrientes mais extraídos foram ferro, manganês, boro e zinco. Segundo Maia (1998) o potássio é acumulado em grandes quantidades nas folhas, pois este nutriente é requerido na absorção do nitrogênio e teor de óleo. Assim, neste trabalho, os nutrientes foram encontrados nos tecidos vegetais produzidos na SC e SCP, na seguinte ordem decrescente, para os macronutrientes: K, N, Ca, S, Mg, P e para os micronutrientes: Fe, Mn, B, Zn, Cu.

Com relação ao total dos macronutrientes da SC, encontrados nos tecidos da menta (Tabela 2), observouse que as concentrações de nitrogênio, potássio, cálcio e o magnésio estão na faixa adequada quando comparadas às concentrações das análises de tecidos citadas por Maia (1998). Para os elementos fósforo e enxofre, os valores foram superiores aos obtidos pelo autor. As quantidades de micronutrientes foram superiores aos valores encontrados por Maia (1998).

Com relação à condutividade elétrica, observou-se que a solução nutritiva concentrada atingiu valores entre 0,88 e 2,06 mS cm-1 e, à medida que avançou o ciclo da planta, esta seguiu uma tendência de redução gradual, de acordo com a absorção de nutrientes. As reposições de nutrientes elevaram a CE, porém não foram o suficiente para elevála até o valor original de 1,99 $\mathrm{mS} \mathrm{cm}^{-1}$.

$\mathrm{O}$ pH da solução nutritiva oscilou desde 5,00 a 7,00, tendo um valor médio de 6,31. Observou-se a tendência da planta de menta em alcalinizar a solução, permitindo assim maior disponibilidade dos nutrientes ao longo do período de cultivo.

Neste estudo, não se verificou sintomas de deficiência nutricional nas plantas de menta cultivadas na solução nutritiva concentrada. Portanto, os valores de $\mathrm{CE}$ e $\mathrm{pH}$ foram adequados para o crescimento das plantas de menta, o que pode ser justificado pela produção de massa seca e óleo essencial obtidos no experimento.

Os valores de CE e pH foram próximos aos citados por Maia (1998) e recomendados por Martinez \& Silva Fi- lho (1997) para cultivos hidropônicos.

O cultivo da Mentha arvensis em solução nutritiva, em sistema hidropônico, alterou o rendimento vegetal de fitomassa e de óleo, bem como a duração do ciclo produtivo permitindo que se fizesse um maior número de colheitas por ano quando comparado com o cultivo em solo.

A solução nutritiva com concentração de $100 \%$ no transplante e reposição de 50\% dos nutrientes quando a condutividade elétrica reduzir 50\% do valor inicial e o espaçamento 0,50 x 0,25 m pode ser recomendada para o cultivo hidropônico de Mentha arvensis, por permitir os melhores resultados de rendimento de fitomassa e óleo quando comparada com as outras soluções testadas ou mesmo com o cultivo em solo.

\section{REFERÊNCIAS}

ADAMS RP. 1995. Identification of essential oil components by gas chromatography/mass spectroscopy. Illinois: Allured Publishing Corporation. 469p.

ALENCAR JW; CRAVEIRO AA; MATOS FJA. 1984. Kovats indici as a presention routine in mass espectro searches of volaties. Journal of Natural Products 47: 890-892.

BARRY C. 1996. Nutrients: The handbook to hydroponic nutrient solutions. Narrabeen, NSW, Austrália, Casper Publications. 55p.

CASTELLANE PD; ARAÚJO JAC. 1995 Cultivo sem solo - hidroponia. Jaboticabal: FUNEP. 43p.

COMISSÃO DE FERTILIDADE DO SOLO RS/SC. 1994. Recomendações de adubação e de calagem para os estados do Rio Grande do Sul e de Santa Catarina. 3.ed. Passo Fundo, SBCS - Núcleo Regional Sul, 1994. 224p.

CORREA JRC; MING LC; SCHEFFER MC. 1991. Cultivo de plantas medicinais, condimentares e aromáticas. Curitiba: EMATER. 162p.

EMBRAPA. Centro Nacional de Pesquisas de Solos. 1999. Sistema Brasileiro de classificação de solos. Brasília: Embrapa. 412p.

FURLANI P.R. 1998. Instruções para o cultivo de hortaliças de folhas pela técnica de hidroponia NFT. Campinas, Instituto Agronômico, 1998. 30p. (IAC. Boletim Técnico, 180).

HOAGLAND DR; ARNON DI. 1950. The water culture method for growing plants without soil. California Agricultural Experiment Station. Circ. 347. Berkeley. 32p.

LONDERO FAA. 2000. Reposição de nutrientes em soluções nutritivas no cultivo hidropônico de alface. Santa Maria: UFSM. 100p. (Tese mestrado). 
MAIA N B. 1994a. Nutrição mineral, crescimento e qualidade do óleo essencial da menta (Mentha arvensis L.) cultivada em solução nutritiva. Piracicaba: ESALQ. 69p. (Tese mestrado).

MAIA NB.1998b. Produção e qualidade do óleo essencial de duas espécies de menta cultivadas em soluções nutritivas. Piracicaba: ESALQ. 105p. (Tese doutorado).

MAIRAPETYAN SK. 1999. Aromatic plant culture in open-air hidroponics. Acta Horticulturae 502: 33-41.

MALUF WR; BOUERI MA; CARDOSO D; BRUGNERA A. 1999. Cultivo e propriedades medicinais da hortelã. Boletim Técnico de Hortaliças 34: 1-3.
MARTINEZ HEP; SILVA FILHO JB. 1997. Introdução ao cultivo hidropônico de plantas. Viçosa: UFV. 52p.

MARTINS ER; CASTRO DM; CASTELLANI DC; DIAS JE. 1994. Plantas medicinais. Viçosa: UFV - Imprensa Universitária. 220 p.

OCAMPOS RK; LAURA VA; CHAVES FCM. 2002. Efeito de diferentes formas de adubação em hortelã rasteira: biomassa e teor de óleo essencial. Horticultura Brasileira 20: suplemento.

SARRUGE JR. 1975. Soluções nutritivas. Summa Phytopathologica 1: 231-233.
SCHMIDT D. 1999. Soluções nutritivas, cultivares e formas de sustentação de alface cultivada em hidroponia Santa Maria: UFSM. 79p. (Tese mestrado).

SIMÕES CMO; SPITZER V. 2003. Óleos voláteis. In: SIMÕES CMO. et al. Farmacognosia: da planta ao medicamento. 5.ed. Porto Alegre/Florianópolis: Editora da UFSC. p. 516.

SINGH VP; SINGH DV. 1989. Accumulation pattern of chemical constituents in mentha species with advance of crop age and nitrogen level. In: INTERNATIONAL SYMPOSIUM ON MEDICINAL AROMATICAND SPICES PLANTS. Acta Horticulturae 188: 187-189. 